\title{
Integrating Inquiry-Based Multimedia Learning Outcomes into Educational Accountability Systems
}

\author{
Steven McGee ${ }^{1}$, Debra Panizzon², John Pegg ${ }^{2}$, \& Bruce C. Howard ${ }^{1}$ \\ ${ }^{1}$ NASA Classroom of the Future, 316 Washington Ave, Wheeling, WV 26003 \\ ${ }^{2}$ University of New England, Armidale, Australia \\ Tel: (304) 243-4308, Fax: (304) 243-2497 \\ Email: mcgee@cet.edu
}

\begin{abstract}
The purposeof this study was to describe and compare two approaches to assessment that were used to identify the growth in students' understandings of planetary processes. These approaches can be integrated into school accountability systems.
\end{abstract}

Keywords: qualitative assessment, quantitative assessment, science education, systemic reform

\section{Introduction}

In the ongoing battle over school budgets in the United States, the word accountability has become the battle cry for those dissatisfied with current education. In fact, most states in the U.S. mandate high-stakes testing of all students as a primary mechanism of school accountability (Kronholz, 1999). Researchers at the NASA Classroom of the Future and the University of New England are directly addressing this issue. Of interest is the need for different approaches to assessment that are aligned to both specific curriculum innovation and high-stakes tests. This paper takes up this theme by describing and comparing two approaches to assessment that were used to identify the growth in students' understandings of planetary processes. Subsequent research will investigate the links between these approaches and high-stakes testing. This study involved Years 5 and 6 students $(n=31)$ in Australia who participated in the Astronomy Village ${ }^{\circledast}$ : Investigating the Solar System ${ }^{\mathrm{TM}}$ project over a two-week period in August 1999. Through Astronomy Village students were transported to a virtual village in Hawaii where they investigated what the surface of Pluto might look like when the first NASA mission arrives in 2015. The program is designed such that a virtual mentor guides students in completing inquiry-based multimedia activities. For a description of the design of Astronomy Village refer to McGee \& Howard (1999).

\section{SOLO: A Framework for Response Coding}

SOLO, an acronym for the $\mathbf{S}$ tructure of the Observed Learning Outcome, is a response model developed by Biggs and Collis in the late 1970s (Biggs \& Collis, 1982). Since that time more than one hundred studies have been undertaken, both to apply and extend the model (Pegg, 1992). In summary, SOLO provides a framework upon which the underlying structure of the answer to a stimulus question can be inferred from the response given. Coding a student's response using the SOLO model depends on two features: mode of thinking and level of response. Of the five modes of thinking, two are relevant to this paper. In the ikonic mode of thinking, a person internalizes actions in the form of images. In this mode the young child develops words and images that can stand for objects and events. In the concrete symbolic mode of thinking, a person thinks through the application of a symbol system such as written language and number systems. This is the most commonly targeted mode of thinking in middleschool and high-school classrooms. Within each mode of thinking, there are three general levels of response. In the unistructural level of response, the student uses only one piece of relevant data and so the response may be inconsistent. In the multistructural level of response, two or more pieces of data are used without any relationships represented between them. No integration occurs of the data and some inconsistency may be apparent. In the relational level of response, all data are now available, with each piece woven into an overall mosaic of relationships. The whole has become a coherent structure. There is no inconsistency within the known system.

\section{Results and Discussion}

Both assessment tasks were administered as pre- and posttests. The first assessment activity instructed students to, "Draw a picture in as much detail as possible showing what you think the surface of Pluto looks like." On the reverse side of the paper, students were then asked " "In as much detail as possible, describe the processes that created the features that you drew." Three researchers using the SOLO model coded these written descriptions. As 
the model is not applicable to visual responses, the drawings were not coded; however, they provided a useful context for interpreting some of the written descriptions.

Most of the student responses were categorized as indicative of the concrete symbolic mode of thinking. We were able to identify two categories of levels of response. The first category was levels of response related to the features drawn in the image. The second category was levels of response related to causal explanations underlying the features drawn in the image. We ranked the levels of response related to causal explanation as more abstract than levels of response related to features. See McGee, Panizzon, Pegg, and Howard (1999) for detailed examples of student responses. A statistical analysis of pre/post performance using a Wilcoxon signed rank test revealed that although a significantly greater number of students improved than declined, the magnitude of the increase was not significant.

The second assessment task was a multiple-choice test already developed for the Astronomy Village summative evaluation effort. Due to the large number of students involved, it was necessary to develop an assessment instrument that could measure inquiry skills in a cost-effective manner. Through a content analysis of Astronomy Village, it was determined that successful students were able to understand complex content as well as draw inferences about planetary processes from images of surface features. Item writers, not involved in the product development, designed multiple-choice items based on this task framework.

Student performance on the Astronomy Village test and the SOLO responses were compared using a Pearson product-moment correlation. To achieve this, the SOLO categories were encoded using 0 for ikonic mode responses and 1 to 6 for the levels in the concrete symbolic mode. The correlations were .438 between the pre SOLO category and the content pretest and .703 between the post SOLO category and the content posttest. These results suggest that the two assessment instruments are measuring highly related but separate constructs. An analysis of pre/post differences provides further evidence that although these constructs are related, they have different developmental trajectories. The mean score for the content test increased from $37 \%$ at the pretest to $54 \%$ at the posttest. This difference was statistically significant $(\mathrm{t}=5.21, p<.01)$. It can be concluded that this implementation of Astronomy Village effectively increased student conceptual understanding and problem-solving.

\section{Conclusions}

In this study, we compared two approaches to classroom assessment. Measuring cognitive reasoning using the SOLO framework provided mixed results from pre to posttest, whereas measuring conceptual understanding and problem solving using the Astronomy Village test showed significant results from pre to posttest. The results also indicate that there is a strong link between the coding of classroom assignments using SOLO and performance on the Astronomy Village test. We believe that these results provide a foundation for research on the relationship between classroom teaching and high-stakes testing. In the next step of our research, we plan to compare performance on the Astronomy Village test with performance on high-stakes testing. This research will allow us to either demonstrate improved performance from participation in Astronomy Village or it will allow us to provide strong evidence that high-stakes testing is not measuring higher-order thinking.

\section{References}

Biggs, J. \& Collis, K. (1982). Evaluating the Quality of Learning: the SOLO Taxonomy. New York: Academic Press.

Kronholz, J. (1999, Oct 29). Colorado School Districts Promise to Make the Grade. Wall Street Journal, p. A20.

McGee, S. \& Howard, B.C. (1999, April). Generalizing activity structures from high school to middle school science. American Educational Research Association annual meeting, Montreal, Canada. [Online] Available: http://www.cet.edu/research/papers/generalizing/main.html. [2000, February 15].

McGee, S. Panizzon, D., Pegg, J., \& Howard, B. C. (1999). Integrating Inquiry-Based Multimedia Learning Outcomes into Educational Accountability Systems. [Online Technical Report] Available: http://www.cet.edu/research/papers/solo/main.html. [2000, February 15].

Pegg, J. (1992). Assessing students' understanding at the primary and secondary level in the mathematical sciences. In J. Izard \& M. Stephens (Eds), Reshaping Assessment Practice: Assessment in the Mathematical Sciences Under Challenge (pp. 368-385). Melbourne: Australian Council of Educational Research. 